\title{
New Scenario to Chaos Transition in the Mappings with Discontinuities
}

\author{
S.V. Naydenov ${ }^{1}$, A.V. Tur ${ }^{2}$, A.V. Yanovsky ${ }^{3}$, V.V. Yanovsky ${ }^{1}$ \\ ${ }^{1}$ Institute for Single Crystals, \\ 60 Lenin avenue, 61001 Kharkov, Ukraine, \\ ${ }^{2}$ Center d'Etude Spatiale Des Rayonnements, C.N.R.S.-U.P.S., \\ 9 avenue du Colonel Roche, 31028 Toulouse, CEDEX 4, France \\ ${ }^{3}$ Institute for Low-Temperatures Physics and Engineering, \\ 47 Lenin ave., 61164 Kharkov, Ukraine
}

\begin{abstract}
We consider a many-parametric piecewise mapping with discontinuity. That is a one dimensional model of singular dynamic system. The stability boundary are calculated analytically and numerically. New typical features of stable cycle structures and scenario to chaos transition provoked by discontinuity are found.

Keywords: mechanisms of chaos, mappings, singularity, bifurcations, stability

PACS: 05.45 -a ; 05.45Ac ; 05.45.Pq
\end{abstract}

\section{Introduction}

It is well known that the dynamical chaos is taken as a basis for the description of complex behavior in different physical systems (see for example [1], [2] and references therein). The transition from regular to chaotic dynamics is typical to dynamical systems with a few number of degrees of a freedom [3]. Even simple dynamic systems can have a complex non-regular behavior [由]. The phenomenon of dynamical chaos is specific not only for classical physics but also for quantum systems [5], [6].

The study of "turbulence" mechanisms for waves and oscillations of different kind processes is one of the more important point for dynamical chaos. To explain the turbulence origins some mechanisms has been proposed: the Landau-Hopf scenario [7], [8]; scenario of Ruelle-Takkens [9] with a strange attractor [10]; crisis with quasi periodicity losses [11]; intermittence transitions [12]; etc. The instability which appears due to the non-linearity and the exponential sensitivity of phase trajectory to initial conditions is crucial for all these cases. The transition to chaos scenario can be a rough one, but the stability loss is going continuously. As a rule the Lyapunov index depends analytically of the control parameter in the bifurcation point.

The more simple and natural models of dynamical chaos are mappings which can be considered as a finite dimensional approximation of real dynamical systems. There are a lot of typical scenario to chaos transition in mappings corresponding to different turbulence mechanisms. For example: period doubling cascade with Fiegenbaum universality 
[13, [14, intermittency [15], strange attractor or repeller appearance [16, [17]. At the same time in the same mapping, but in different control parameter domains, one can observe different kinds of chaos transition. One dimensional and two dimensional one-parametrical mappings are widely used, such as: Bernoulli shift [18] and "saw" [19], logistic mapping [20], Ulam's "tent" [21], twist transformation [22], "backer" transformation [23], Henon's transformation [24], Arnold's "cat" [25] and "standard" Chirikov mapping [26] etc. The one-dimensional continuous mappings and dynamical systems with one control parameter are studied well enough (see for ex. [27]-29]). Bifurcations in bi- and many parametric families are less studied than others. The instability and the chaos transition are generally connected with a smooth non-linearity, a non-monotonic behavior and with a mapping ambiguity. Each of this factors is necessary, but not sufficient conditions for the loss of a stability. The dynamics of mappings with singularities has its special features. Such mappings appear during the description of impact oscillator [30]-[31], during the modeling of noise influence on information capacity [32]-[33], during the research of different dynamic systems with discontinuous characteristics.

The role of such particularities as a break value in one-dimensional discontinuous mapping of interval has not been studied in details, especially for a case, when the break value is less than the interval length (non-overall break). At the same time, the topological discontinuity and stretch non-linearity together lead to some new particularity of the dynamical behavior. In this paper we are considering such features for the simple piecewise linear mapping case with discontinuity.

The most important new feature of mappings with discontinuity as it will be shown latter is a new bifurcation of cycle deleting, which we will call "put-out" bifurcation. This bifurcation is different from other ones in smooth dynamical systems by the jumplike variation of multiplicator (mapping Jacobian). As a result of such a bifurcation the stable limit cycle can simply disappear. Afterwards, the system can get immediately in the global chaos state. Roughly speaking the "put-out" bifurcation appears as a result of coincidence of one cycle point with break coordinates. The mapping which we consider is two-parametric and that is why it has a rich enough dynamic, both regular and chaotic.

\section{Mapping with a discontinuity}

Let us consider the family of piecewise linear mappings for the interval $I=[0,1]$ :

$$
x \rightarrow f(x)=\sum_{\sigma} f_{\sigma}(x) \chi_{\sigma}(x) ; f_{\sigma}(x)=k_{\sigma} x+b_{\sigma},
$$

where $\chi_{\sigma}$ - is indicator of the natural partition of phase space $I=\bigcup_{\sigma} I_{\sigma}$ for function $f$ monotony (linearity) intervals. We will restrict our study to bimodal (with two critical points) mappings with one break only. For our mapping we have:

$$
I_{\sigma}: I_{1}=[0, a] ; I_{2}=[a, 1 / 2] ; I_{3}=[1 / 2,1-a] ; I_{4}=[1-a, 1],
$$

where $x=a$ and $x=1-a$ are critical points; $x=c=1 / 2-$ is a break coordinate. Mapping parameters

$$
\left\{\begin{array}{l}
k_{1}=k_{4}=\mu_{1}=\frac{A}{a} \\
k_{2}=k_{3}=\mu_{2}=\frac{2(E-A)}{1-2 a}
\end{array} \quad ;\left\{\begin{array}{l}
b_{1}=0 ; b_{4}=1-\frac{A}{a} \\
b_{2}=\frac{A-2 a E}{1-2 a} ; b_{3}=1+\frac{A-2(1-a) E}{1-2 a}
\end{array}\right.\right.
$$


include a critical value $A, 0 \leq A=f(a) \leq 1$ and a break height $E=1+\varepsilon / 2$, where $1 / 2 \leq E \leq 1$ (with a gap $0 \leq \varepsilon \leq 1$ ). The plot of the mapping is shown on fig. 1 .

The mapping (1)-(3) with symmetry (involution) $G$ is reversible mapping

$$
f \circ G=G \circ f \Leftrightarrow f(x)=1-f(1-x) ; G(x)=\tilde{x} \stackrel{\text { def }}{\equiv} 1-x,
$$

where $G \circ G=i d$; $\circ$ is mappings composition, $i d$-identical transformation. The reversible systems are important from physical point of view and a lot of papers treats this subject [34]-36], etc.

The mapping family (1) is three parametric $f=f_{\Omega}$, but further it will be enough to consider a two parametric case with $a=$ const. It seems convenient to choose as independent coordinates on $\Omega_{a}$ parameter surface a set of $(A, E)$. A necessary but not sufficient condition for chaotic behavior is a stretching condition so as to at least one of the multiplicators modules $\left|\mu_{1,2}\right|$ does exceed one. Let us choose $A>a \Leftrightarrow \mu_{1}>1$. Because of reversibility we can consider that $0<a \leq 1 / 2$. The mapping becomes non-monotonous and non- one-to-one at $\mu_{2}<0$, i.e. lower $(E<A)$ than the diagonal $\Delta(A=E)$ of the parametrical space.

The mapping iteration dynamic as well as its bifurcation must be considered in total space $Z=I \times \Omega$. For one dimensional system we can consider only the section of the space $Z$ (stability diagram) and bifurcation diagrams on hyper planes $I \times D(D-$ the value of control parameter in one parametric family of mappings given by equation $\Phi(A, E ; a)=0)$. This last case concerns also bifurcation diagrams in coordinates $(x, A)$, $E=$ const; $(x, E), A=$ const and $(x, \Delta)$, where $\Delta=A \equiv E$ is a parameter along the diagonal of surface $\Omega_{a}$. Periodical trajectories (cycles) play a key role for the dynamics of one dimensional mappings. Their structure and stability determine the regular and the chaotic dynamic of a system.

\section{Cycles structure}

Coordinates of arbitrary cycle $C=\left\{x_{i}\right\}_{i}, i=0, \ldots, p-1$ are defined by equations $f^{p}\left(x_{i}\right)=x_{i}$, with conditions $f^{k}\left(x_{i}\right) \neq x_{i}, k=1, \ldots, p-1$. All cycle points are different $x_{i} \neq x_{j}, i \neq j$ due to the uniqueness of the phase trajectory. Point cycle numbering can be changed cyclically.

Each cycle $C$ is given by a set of characteristic parameters such as a period $p \geq 2$, a kind $(n, k)(p=n+k)$ and itinerary $W$ (topological type). The kind of cycle is determined as a number of points that belong to monotony segments in neighborhood of discontinuity, i.e. to segments $I_{2}$ and $I_{3}$ of nature partition. The cycle itinerary $W(C)=\left\{\sigma\left[x_{i}\right]\right\}_{i}$ is defined by the cyclic sequence of its point addresses in nature partition $I$, where $\sigma[x]=\sum_{\sigma} \sigma \chi_{\sigma}(x)$ is an address which has one of four possible values $\sigma=1,2,3,4$, for example. In addition, the points itinerary of this cycle $W\left(x_{i}\right)=\left\{\sigma\left[f^{k}\left(x_{i}\right)\right]\right\}_{k}$ is also important. They are formed by different permutations of the given itinerary $W\left(x_{0}\right)$ with a fixed initial point $x_{0}$. It is necessary to differ cycle itinerary from point one.

The cycle stability is determined by multiplicators $\mu(x)=d f(x) / d x$ in each of its points. According to chain rule of differentiation we have:

$$
\mu(C)=\mu^{(p, k)}\left(x_{i}\right)=\prod_{k=0}^{p-1} d f\left(f^{k}\left(x_{i}\right)\right) / d x=\prod_{k=0}^{p-1} \mu_{\sigma\left[x_{k}\right]}=\mu_{1}^{p-k} \mu_{2}^{k}
$$


where the cycle multiplicator does not depend of chosen point $x_{i}$. It is convenient to introduce the multiplicator module $m=|\mu|$. For stable $C^{s}$ cycle $0 \leq m\left(C^{s}\right)<1$, for unstable $C^{u}$ cycle $m\left(C^{u}\right)>1$. On the stability limit $m(C)=1$ and additional analysis of stability is required.

Let us describe in details the cycle structure of our mapping. In accordance with the symmetry (플 all cycles can be dived into symmetric and non symmetric ones. For non symmetric cycles ( $\alpha$-type) we have $C_{\alpha} \neq G\left(C_{\alpha}\right)=\tilde{C}_{\alpha}$. The symmetric cycle ( $\beta$-type) coincides with its dual one $C_{\beta}=G\left(C_{\beta}\right)=\tilde{C}_{\beta}$. Because of reversibility equation $\tilde{\tilde{C}}=C$ is valid for any cycle. The non symmetric cycles of reversible mappings appear as dual pairs only $C_{\alpha}$ and $\tilde{C}_{\alpha}$. Dual cycles multiplicators coincide $\mu(C)=\mu(\tilde{C})$. This is why it is enough to show on stable cycle diagram only one of dual cycle. On bifurcation diagram dual cycles are symmetric with respect to $x=1 / 2$ right line.

Cycles of the same period $p$ constitute $p+1$ non-overlapping (non crossing) classes in accordance with possible values $k=0, \ldots, p$. For the family of piecewise mappings this partition is simple: each class consists of only one specimen (with the accuracy for reversibility $C \rightarrow \tilde{C}$ ). The kind $k=1$ or $k=2$ corresponds to non symmetric or symmetric cycle correspondingly. The piecewise mappings cycles including the break ones have some particularities. The topological type $W(C)$ determines all the cycle $\mathrm{C}$ without ambiguity. The reason is that the linear equation system for definition of cycle points may have only one unique solution. In addition, for the same reason there are no two different cycles for the same period $p_{1}=p_{2} \Rightarrow C_{1}=C_{2}$. Cycles have following isomorphic descriptions

$$
C\left(p ; x_{0}\right) \leftrightarrow W(C) \leftrightarrow\{\mu(C) ; k\}
$$

All stratified cycle structure may be shown as

$$
\begin{gathered}
C=C_{\text {asymm }} \oplus C_{\text {symm }} ; C=C^{\text {stab }} \oplus C^{\text {nonstab }} ; \\
C^{\text {stab }}=C_{\text {asymm }}(k=1) \oplus C_{\text {symm }}(k=2) ; \\
C=\bigoplus_{p=2}^{\infty} C(p)=\bigoplus_{p=2}^{\infty} \bigoplus_{k=0}^{p} C(p, k)=\bigoplus_{W} C(W),
\end{gathered}
$$

where $\oplus$ means the direct sum of subspaces. It is shown schematically on fig. 2. Mapping is bimodal, that is why stable cycles higher then $k \geq 3$ kind do not exist. Non-symmetrical stable cycles of kind $k=1$ can be of even and odd period $p \geq k$. Symmetric stable cycles have kind $k=2$ and even period only. Stable cycles of zero $k=0$ do not exist in such a system. Stable cycles of different itinerary can not coexist. In the contrary the coexistence of different type of non stable cycles is not forbidden. Outside of stability area all non symmetrical stable cycles are situated below the diagonal $A=E$. Symmetrical stable cycles of the same type are filling two one-link domains on parameter surface separated by stability area. This is due to the monotony of the mapping with $A>E$. All the cited regularities are proved directly or verified by numerical experiments fig. 3, fig. 4 .

\section{Put-out bifurcation}

Reconstruction of cycle structure is determined by "put-out" bifurcation which is an important point for dynamic of system with break. The "put-out" bifurcation means 
the reconstruction leading to sudden disappearance or appearance of cycles. In a general case we will define the "put-out" bifurcation as a bifurcation accompanied by jump like changing of mapping multiplicator (Jacobian) in any point of cycle. It means

$$
C_{1} \rightarrow C_{2} ; \quad\left|\mu\left(C_{1}\right)\right| \neq\left|\mu\left(C_{2}\right)\right| .
$$

The breaking makes the essential difference between the "put-out" bifurcation and usual continuous bifurcation cycles including "fork" bifurcation with doubling of the period and disappearance of fixed point (cycle) for the intermittency on the stability threshold.

Let us consider the topological reasons of "put-out" bifurcation. "Special" trajectories with initial points in the critical value of mapping or discontinuity are very important for mapping with singularities. With some values of parameter they become periodical (cycles). Special cycles give Markovian partitions for space phase. The dynamic of all others cycles must be coherent and is controlled by these partitions. One of the point of special cycle may coincide with any point of usual one when control parameter are changing. In this case the jump-like changing of the multiplicator takes place and the "put-out" bifurcation appears. Special cycles coordinates of different type are defined, for our case, by finite number of linear equation. Special cycles are forming lines of co-dimension one on the plane of parameters. These lines represent limits of "put-out" bifurcation.

The conditions of "putting-out" correspond to the cyclicity of a special trajectory with itinerary $W\left(f^{n}(\hat{x})\right)$ of all its points $f^{n}(\hat{x})$ following. The points correspond to itinerary $W$ of the cycle of period $p$ under consideration

$$
f^{p-n}\left(f^{n}(\hat{x}) \mid W\left(f^{n}(\hat{x})\right)\right)=\hat{x} ; f^{p}(\hat{x})=\hat{x} ; \hat{x}=\{a ; c\}
$$

where by $a$ and $c$ the coordinates of critical points and the points of discontinuity are represented.

The borders of "put-out" bifurcation for 2-parametric $(a=$ const) family (1) should be put down as:

$$
\left\{\begin{array}{l}
f^{p-n}\left(f^{n}(a) \mid W\left(f^{n}(a)\right)\right)=a \\
f^{p-n}\left(f^{n}(1-a) \mid W\left(f^{n}(1-a)\right)\right)=1-a \\
f^{p-n}\left(f^{n}(1 / 2 \mp 0) \mid W\left(f^{n}(1 / 2 \mp 0)\right)\right)=(1 / 2 \mp 0)
\end{array}\right.
$$

The itineraries for different points of the same cycle are different, $W\left(f^{k_{1}}\left(x^{*}\right)\right) \neq$ $W\left(f^{k_{2}}\left(x^{*}\right)\right)$. Each composition $f^{p}$ has $N_{p}$ one-to-one branches. So all the $N_{p}=4 p$ of the conditions (10) should be regarded as independent. For stable cycle s or for symmetric cycles the dual conditions, differing by the change of form (10), coincide. Moreover, for stable cycles all the conditions of every type are equivalent, so the following independent conditions are left:

$$
f^{p}(a \mid W(a))=a ; f^{p}(1 / 2 \mid W(1 / 2))=1 / 2 .
$$

As an example, let us cite the borders of "putting-out" for 24, 2431 and 2441-cycles (the itineraries are mentioned). The first of the conditions (11) gives $A=A_{24}=1-a$; $A=A_{2431}=A_{2441}=\frac{1}{2}\left[1+\sqrt{1-4 a^{2}}\right]>A_{24} ;$ and the second one gives $E=E_{24}(A)=$ $E_{2431}(A)=1-\frac{a}{2 A}$ and $E=E_{2441}(A)=1-\left(a^{2} / A^{2}\right)(1-a /(2 A))>1 / 2$. The equations $A=A(a)(a=$ const $)$ and $E=E(A)$ obtained define the lines of "putting-out". In the 
general case they are straight segments $(A=$ const $)$ and algebraic curves $E-E_{W}(A)=0$ (of $p-1$ order for a non-symmetric cycle with period $p$ ).

Markovian partitions of hyperbolic mappings are everywhere dense [37], [38]. The analogous thing is true for the mappings (10), corresponding to the "putting-out" of unstable cycles of arbitrary high order $p=2,3, \ldots, \infty$. Let us note that this lets one calculate the (statistic) invariant measure of mapping almost everywhere in chaotic region. The rigorous solution is obtained at the parameters corresponding to every intersection point of curvilinear coordinate lines of the bifurcational web of the borders of "puttingout". Whereas the coordinate families mentioned are everywhere dense on the parametric plane.

"Put-out" bifurcation is a characteristic feature of not only one-dimensional systems of the type (1), but also of multi-dimensional and multi-parametric nonlinear systems with and without breaks. In discontinuous systems, that, according to Baire, make up a set of the first category (see, for ex., [39]) in the space of arbitrary dynamic systems - this is a typical feature. In continuous systems, the presence of critical points (singularities) is required. The traces of "putting-out" can be found in every discontinuous mapping. The new mechanisms of transition to chaos, different from those known before, will be connected with "putting-out". The necessary condition of chaotic behavior is the loss of stability. At that, in continuous systems, cycles lose stability in a continuous way. In discontinuous systems, the mechanism of spontaneous loss of system stability (in large), at "putting-out" of a stable cycle with non-unit module of the multiplier, appears. Such a stable cycle can be situated far from its border of stability, $|\mu|<1$.

\section{$5 \quad$ Stability}

Conditions for cycle stability $\mu_{1}^{p-k}\left|\mu_{2}^{k}\right|<1$ in variables $0<A \leq 1$ and $0<E \leq 1$ $(a<1 / 2$ fixed $)$ has the form

$$
\left(\frac{A}{a}\right)^{p-k}\left|\frac{2(A-E)}{1-2 a}\right|^{k}<1 .
$$

It corresponds with stability boundaries of cycle $(p, k)(p$-period, $k$-kind given by following equations

$$
E=E_{ \pm}^{p, k}(A)=A\left\{1 \pm\left[a^{\frac{p-k}{k}}(1 / 2-a)\right] A^{-\frac{p}{k}}\right\}
$$

for $1 \geq E>A \geq 1 / 2$ or $1 / 2 \leq E<A \leq 1$. Let us note, that stability boundaries for symmetric stable cycles may coincide with a part of boundary with the "put-out" of non-symmetric and non-stable cycles (see fig. 3).

With fixed $p$ and $k$ stability boundaries (13) are algebraic curves of $p+k$ order. They limit stability zone in the square $(1 / 2 \leq A \leq 1 ; 1 / 2 \leq E)$. The curve $E=E_{+}(A)$ is convex (down), $E=E_{-}(A)$ is concave (from above). On the diagonal $A=E$ of surface $(A, E)$ every kind of unstable cycle is "super" stable

$$
A=E \quad \Rightarrow \quad m(C)=0
$$

The surface of limit admissible zone of stability decreases with the increasing of period $p$ i.e. $S\left(p_{1}\right)>S\left(p_{2}\right)$ with $p_{1}<p_{2}$. (The real zone of stability comparing to the limit one 
is less important due to cycles "put-out"). It follows from the monotonically decreasing dependence $E_{ \pm}=E_{ \pm}(A ; p, k)$ from $p$ (derivative $\left.\partial E_{ \pm} / \partial p<0\right)$ and is in accordance with the fact that with the increasing of $k$-kind fixed stable period cycles their stability decreases. Numerical experiments still confirm this conclusion when taking into account "put-out" cycles. The largest area on the stable cycles diagram is possessed by the most stable stable cycles of 23 type, then stable cycles 24 and 2431 and so on.

\section{Chaotisation mechanisms}

The chaotisation mechanism depends on the way of system stability loss. In continuous system this transition is continuous. During it, the last stable cycle's left multiplicator module passes over the unit value. In systems with breaks, as mentioned above, a cycle can be entirely "put out " up to the loss of stability. (Non-symmetric cycle can be "put out" only preserving the system stability, i.e. by appearing of a new and already symmetric stable cycle. The cascade of period doubling (see below) corresponds to it.) "Putting out" a still stable cycle can result in stability loss of the whole system. This is the spontaneous chaotisation mechanism.

A typical feature of continuous one-dimensional mappings is the cascade of period doubling. In it, at the same time with the smooth loss of stability by some cycle, another stable cycle with doubled period arises. Cycle multiplier is changing continuously. The cascade of doubling is a part of Sharkovsky series [27] of constant mapping for cycles ordering by period. In mappings with breaks the cascade of doubling is also possible. But it is caused not by constant stability loss in one of the cycles, but by its "putting out" and replacing it by other stable cycle. There is a jump between the multiplier of the initial cycle and that of the cycle with double period. Bifurcation of such type is called "putting out" with doubling.

The rebuilding during which a stable cycle of a certain type is replaced by a cycle of some other type but the same period is forbidden in continuous systems, because this should be accompanied with a jump of cycle multiplier. In discontinuous systems such type of stable cycles bifurcation is allowed. It is called "putting out" with period preserving. Let us point out that the stable cycle stability is preserved in this case.

"Putting out" of unstable cycles with preserving or changing of the period is possible either in continuous or discontinuous systems. The permissibility of isolated cycles multiplier jump is guaranteed by everywhere dense in fill of the chaotic system phase space with other periodic trajectories. (The averaged multiplier of the mapping near such bifurcation point is continuous.) "Putting out" of the unstable cycles influences the stochasticity procedure. The density of invariant distribution, metric entropy and so on are measured by the jump.

The three types of bifurcation mentioned belong to mapping stable cycles. The route of every stable cycle can be represented as $W\left(C_{\alpha}^{s}\right)=s u U$ and $W\left(C_{\beta}^{s}\right)=s u U \tilde{s} \tilde{u} \tilde{U}$, where $s=2,3$ - the address of cycle points on the components adjacent to the break; $U$ is a sequence of other $u=4,1-$ addresses; $\tilde{s}=3,2$ and $\tilde{u}=4,1$ are dual addresses. Such representation is imposed by the exclusion of $k \geq 3$-type stable cycles in the structure

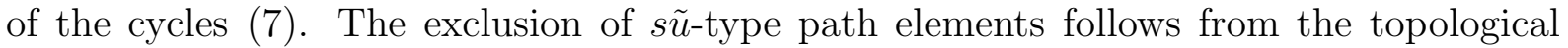

dynamics of the cycles. With the use of these symbols the types of stable cycles "putting 
out" can be represented as:

$$
\begin{gathered}
\text { 1) } C_{\beta}^{s} \leftrightarrow C_{\alpha}^{u} ; m\left(C_{\beta}^{s}\right)<1<m\left(C_{\alpha}^{u}\right) ; \\
\text { 2) } C_{\alpha}^{s} \leftrightarrow C_{\beta}^{s} ; W\left(C_{\beta}^{s}\right)=W\left(C_{\alpha}^{s}\right) W\left(\tilde{C}_{\alpha}^{s}\right) ; \\
m\left(C_{\beta}^{s}\right)=m^{2}\left(C_{\alpha}^{s}\right)<m\left(C_{\alpha}^{s}\right)<1 ; p_{\beta}=2 p_{\alpha} ; \\
\text { 3) } C_{\beta}^{s} \leftrightarrow C_{\alpha}^{s} ; W\left(C_{\beta}^{s}\right)=s u U \tilde{s} \tilde{u} \tilde{U} ; \\
W\left(C_{\alpha}^{s}\right)=s u U u \tilde{u} \tilde{U} ; m\left(C_{\beta}^{s}\right)<m\left(C_{\alpha}^{s}\right)<1 ; p_{\beta}=p_{\alpha} .
\end{gathered}
$$

This is schemed on fig. 5. At spontaneous chaotisation the symmetric stable cycle disappears. At the same time, far from it (in the phase space) an asymmetric unstable cycle of the same period and corresponding route $(\tilde{s}$ replaced by $\tilde{u})$ is situated. So spontaneous chaotisation corresponds to global bifurcation of discontinuous mapping stable cycles "putting out".

The explicit nature of the types of "putting out" under consideration can be observed on the diagram of mapping stability (11). It is numerically obtained at $a=0.4$ and shown in fig. 3 and fig. 4 . The repetition of bifurcations with stable cycle period doubling and preserving leads to cascades of doubling. Every cascade is generated by an "irreducible" stable cycle. Together they combine into a ladder of cycles. Irreducible are cycles of the type: $24 ; 244 ; 24 \ldots 4$ (they make the main section of the ladder); $244144 ; 24414 ; 244141$ and others (they fill the windows between the cascades of the main series). The regions of stable cycles are limited by the lines of stability from above and below. The ladder of stable cycles is of fractal character. Spontaneous chaotisation takes place at transition from one of its steps (from the region of stable symmetric cycle) into the chaotic region (beyond the limits of the next step of doubling cascade). For example, the transition from 23-drains zone to the region above the step that includes zones of 24- and 2431- stable cycles. This transition takes place at $A=A_{23}=A_{24}=1-a$ and $(2-3 a) / 2(1-a)<E<(3-4 a) / 2$ or $(1-2 a) /(1-a)<\varepsilon<2(1-2 a)$. For the cycles of a higher period, similar transitions are possible not only above, but also below the diagonal $A=E$.

\section{Transitions to chaos}

Let us briefly discuss the features of the dynamics under consideration. The transition to chaos in a singular system can take place with different scenarios. They correspond to different one-parametric deformations in the family of mappings. On the parametric plane, such deformations define the motion along some curves. The change of modes corresponds to the intersection of the "dynamic state" line with bifurcational curves and the curves of stability. The way of transition to chaos depends on the three elementary processes, connected with the change of stability. This is an ordinary stability loss of the cycle and the "putting out" of a stable cycle. "Putting out" of a stable cycle can lead to stability loss for the system in general or doubling of stable cycle period (and a step-wise decrease of its stability). The doubling is connected with the break gap variations at the fixed amplitude is $1 / 2<A<1$, and the spontaneous chaotisation is connected with the amplitude variations of the mapping with a gap $\varepsilon \neq\{0,1\}$. 
In continuous systems, chaos is caused by their non-linearity and stretching (in onedimensional mappings). In singular systems the gap iterations dynamics is essential. Filling the phase space, the gaps can preserve or ruin the regularity of the main phase trajectories or make them "double". So, singularity shows itself as strong "non-linearity". Its distinguishing feature is the uneven character of the processes that take place when the main parameters of the trajectories, for instance, the multipliers of the cycles break in the points of bifurcation. The nonlinearity and "putting-out" are closely connected, because they both are absent in a linear system. "Putting-out" of unstable cycles is typical for discontinuous systems and can take place in continuous ones (trajectories thinning). In this case, the singularity directly influences the internal properties of chaos.

Among chaotisation scenarios for discontinuous mapping one can point out the following transitions: symmetric stable cycle - (spontaneous) chaos; non-symmetric stable cycle - (ordinary) chaos; the cascade of doubling by "putting-out" - chaos; stable cycle chaos - regularity windows (doubling cascades) - chaos ("putting-out alternation"). All of them can be easily identified in the cycles diagram fig. 4 - fig. 5. The main role in the chaos development is played by the stable cycles "putting-out" caused by the singularity of the system.

\section{Summary}

So, on the example of a piecewise-linear mapping with a break the typical features of regular and chaotic dynamics of singular dynamic systems are determined. The cycles "putting-out" bifurcation for continuous and discontinuous mappings is obtained. The "putting-out" conditions are obtained and their connection with Markovian phase space partitions, generated by the system properties (critical points and breaks) is stated. For discontinuous mappings, three types of stable cycles "putting-out" are distinguished - with the stability loss at "putting-out" of the whole stable cycle (spontaneous chaotisation); preserving stability and doubling or preserving of a cycle's stable period at the change of symmetry character. The first case corresponds to the new chaotisation mechanism of singular dynamic systems. The second one corresponds to the development of period doubling cascades, caused not by the stability loss of the cycles, but by their "putting-out" from the full structure of mapping cycles. The cycle multipliers, and so their parameters, defining the chaoticity of the system (Lyapunov index etc.), in all other types of "puttingout" are measured by a step.

The research of "putting-out" bifurcation and the spontaneous mechanism of stability

loss will help us find out fundamental regularities of the deterministic chaos is arbitrary singular dynamic systems.

\section{References}

[1] H.G. Shuster, Deterministic Chaos, (Springer, Heidelberg, 1982).

[2] P. Cvitanović, R. Artuso, R. Mainieri, G. Tanner, G. Vattay, Classical and Quantum Chaos, avaliable on site (Internet): www.nbi.dk/ChaosBook/ , (Niels Bohr Institute, Copenhagen, 2002). 
[3] A.J. Lichtenberg and M.A. Liberman, Regular and Stochastic Motion, (Springer, New-York-Heidelberg-Berlin, 1983).

[4] R.M. May, Nature, 261, 459 (1976).

[5] M.G. Gutzwiller, Chaos in Classical and Quantum mechanics, (Springer-Verlag, New York, 1990).

[6] H.-J. Stockmann, Quantum Chaos: An Introduction, (Cambridge, University Press, New York, 1999).

[7] L.D. Landau, Docl. Acad. Sci. of USSR 44, 339 (1944).

[8] E. Hopf, Commun. Pure and Appl. Math. 1, 303 (1948).

[9] D. Ruelle, F. Takkens, Comm. Math. Phys. 20, 167 (1971).

[10] E.N. Lorenz, J. Atmos. Sci. 20, 130 (1969).

[11] C. Grebogi, E. Ott, J.A. York, Physica D 7, 73 (1983).

[12] P. Berge, Y.Pomoeau, C. Vidal, Order Within Chaos, (Wiley, New York, 1986).

[13] J. Guckenheimer, Invent. Mathem. 39, 165 (1977).

[14] M.J. Feigenbaum, J. Stat. Phys. 19, 25 (1978).

[15] P. Manneville, Y. Pomeau, Phys. Rev. Lett. A 75, 1 (1979).

[16] E. Ott, Rev. Mod. Phys. 53, 655 (1981).

[17] P. Gaspard, Chaos, Scattering and Statistical Mechanics, (Cambridge Univ. Press, Cambridge, 1997).

[18] P. Billingsley, Ergodic Theory and Information. (Wiley, New York, 1964).

[19] A. Renyi, Acta Math. Acad. Sci. Hungary 8, 477 (1957).

[20] S. Grossmann, S. Thomea, Z. Naturforsch. 32A, 1353 (1977).

[21] S.M. Ulam, J. von Neuman, Bull. Amer. Math. Soc. 53, 1120 (1947).

[22] J. Moser, Stable and Random Motions in Dynamical Systems, (Prinston University Press, New Jersey, 1973).

[23] E. Ott, Chaos in Dynamic Systems, (Cambridge Univ. Press, Cambridge, 1993).

[24] M. Henon, Commun. Math. Phys. 50, 69 (1976).

[25] V. Arnold, A. Avez, Ergodic Problems of Classical Mechanics, (Benjamin, New York, 1968; Addison-Wesley, Redwood City, 1989).

[26] B.V. Chirikov, Phys. Rep. 52, 463 (1979).

[27] A.N. Sharkovskii, Ukrainian Math. J. 16, 61 (1964). 
[28] T.Y. Li, J.A. York, Am. Math. Monthly 82, 985 (1975).

[29] P. Collet, J.P. Eckmann, O.E. Landford, Comm. Math. Phys. 76, 211 (1980).

[30] W. Chin, E. Ott, H.E. Nusse, C. Grebogi, Phys. Rev. E50, 4427 (1994).

[31] H. Landa, Physica D 82, 117 (1995).

[32] E.M. Bollt, Y.-C. Lai, C. Grebogi, Phys. Rev. Lett. 79, 3787 (1997).

[33] K. Zyczkowski, E.M. Bollt, Physica D 132, 393 (1999).

[34] V.I. Arnold, M.B. Sevryuk. Oscillations and bifurcations in reversible systems, in: Nonlinear phenomena in plasma physics and hydrodynamics (ed. R.Z. Sagdeev) Mir, Moskow, 1986, 31-64.

[35] A. Politi, G.L. Oppo, R.Badii, Phys. Rev. A 33, 486 (1986).

[36] J.A.G. Roberts, G.R.W. Qwispel, Phys. Rep. 216, 1 (1992).

[37] Ya.G. Sinai, Functional Anal. Appl. 2, 245 (1968).

[38] R. Bowen, Equilibrium States and the Ergodic Theory of Anosov Diffeomorphisms, (Springer Lecture Notes in Math., v. 470, 1975).

[39] J.C. Oxtoby, Measure and Category, (Springer-Verlag, New York, 1971). 


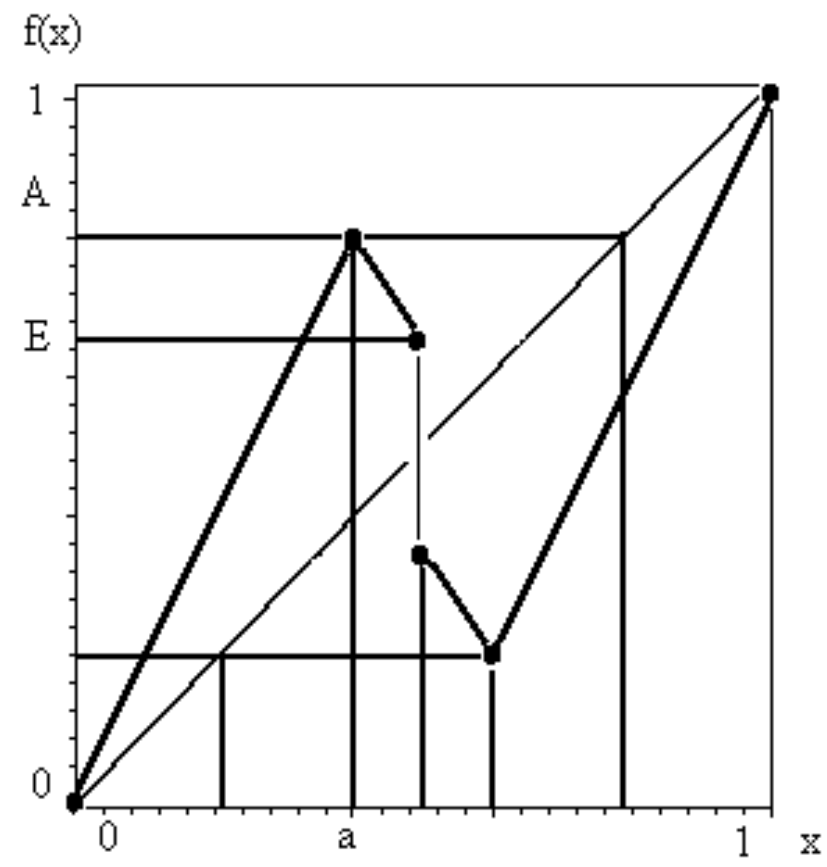

Figure 1: Piecewise-linear mapping with a break.

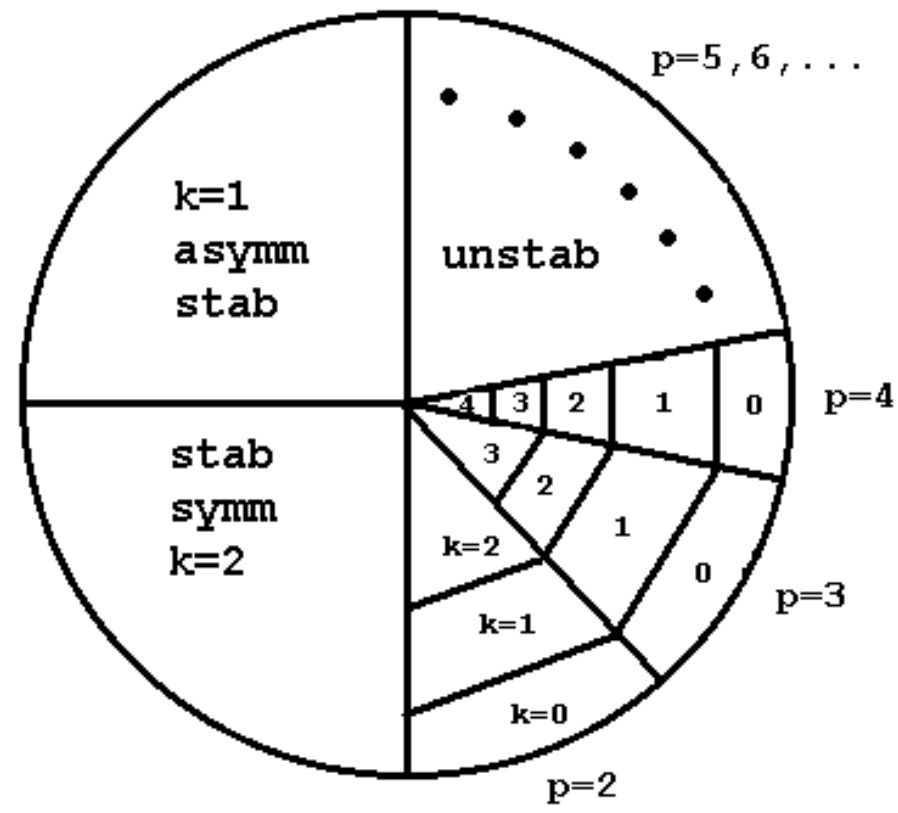

Figure 2: The scheme of stratified structure of the cycles. 

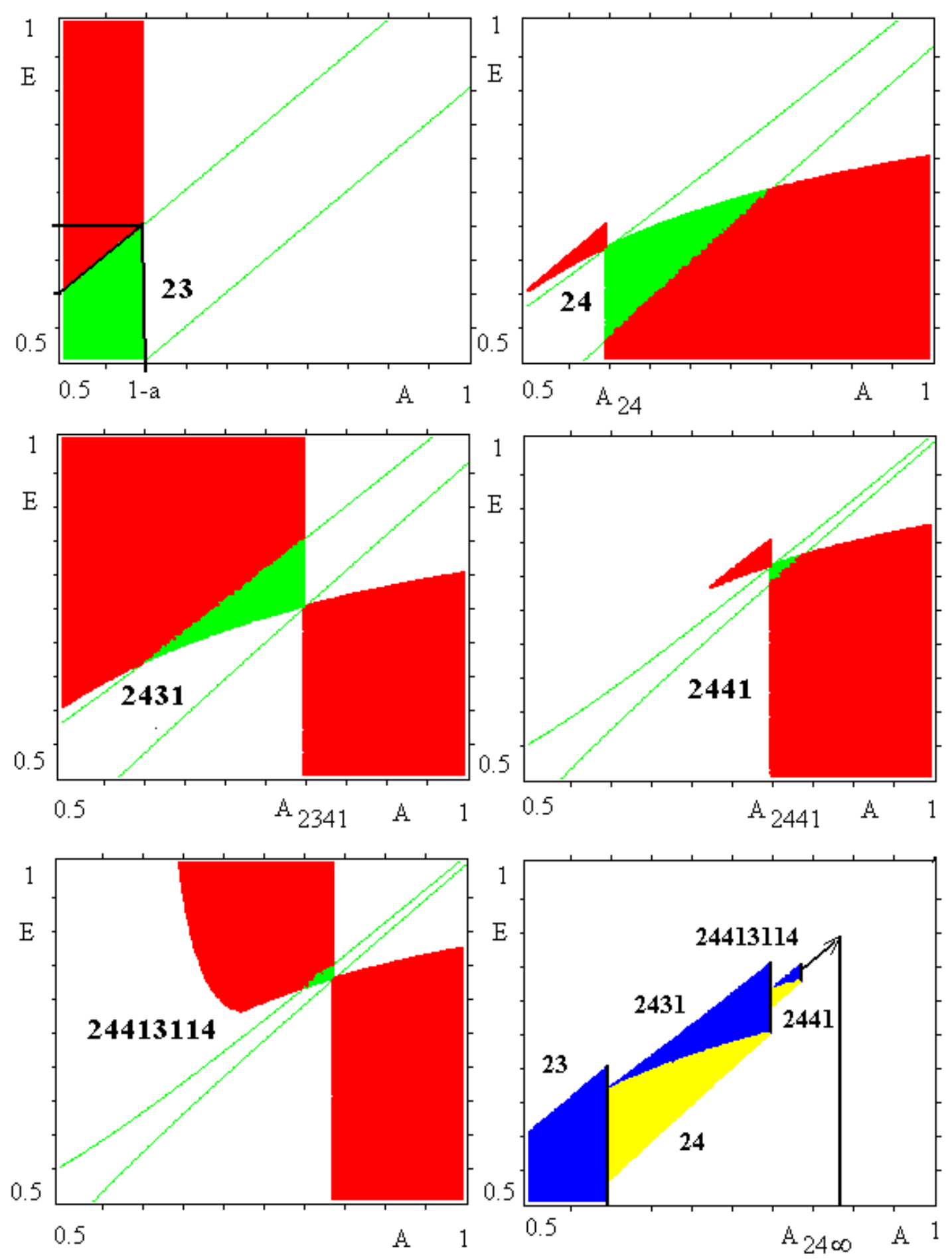

Figure 3: The cycle diagram in the stability zone of the mapping. On the plane of the control parameters (A, E) stable and unstable cycles are mapped with the following coinciding routes: $\mathrm{W}=23 ; \mathrm{W}=24 ; \mathrm{W}=2431 ; \mathrm{W}=2441 ; \mathrm{W}=24413114$ and the full 24cascade of doubling of the stable cycles experiencing "putting-out" bifurcation with the change of the symmetry type. 

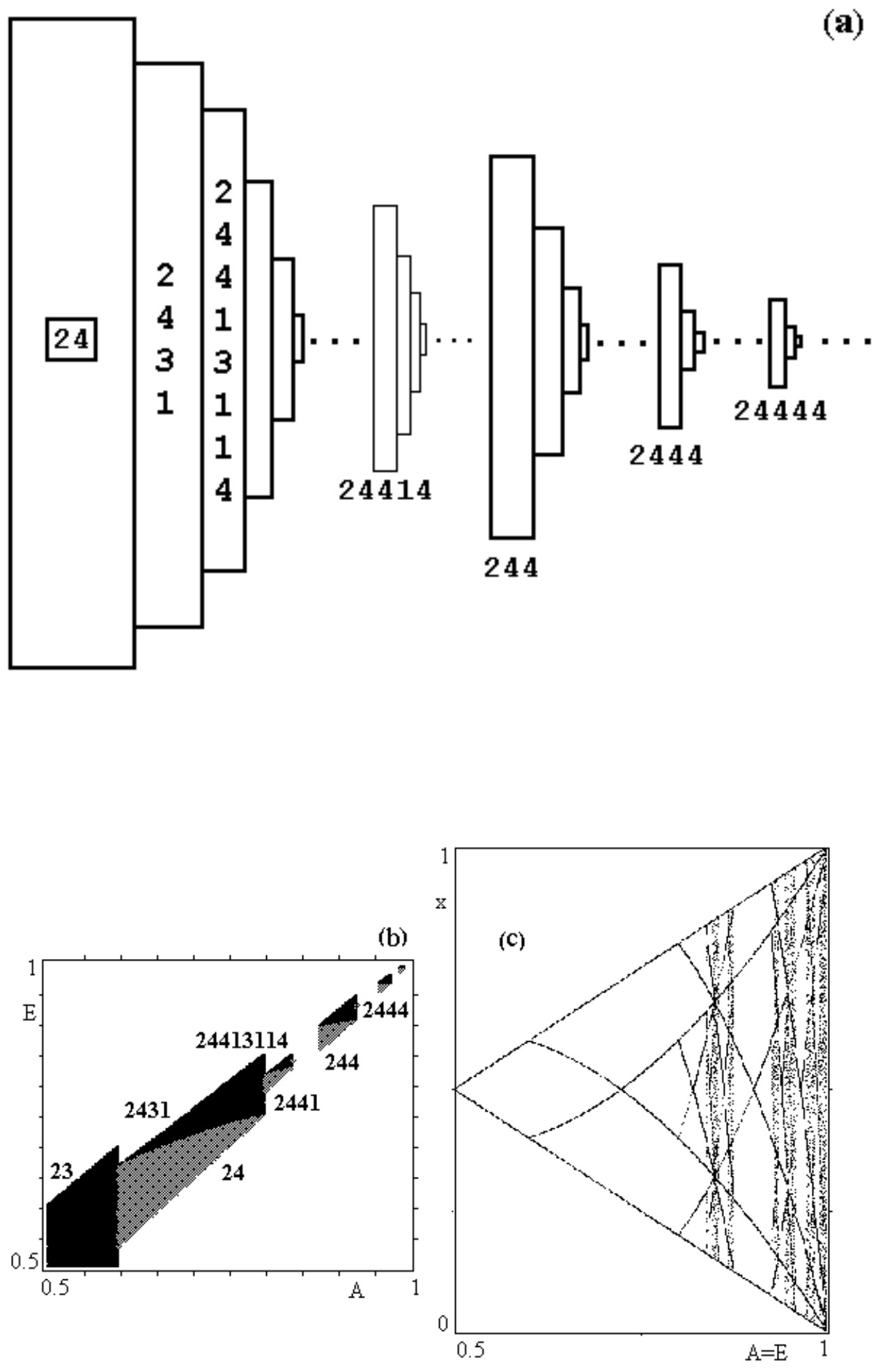

Figure 4: The diagram of stable cycles: a) a telescopic structure (schematically); b) the main $(24 ; 244 ; 244 ;)$ series of a telescopic structure of the stable cycles; c) the skeleton of some irreducible stable cycles, "generating" the cascades with period doubling. 


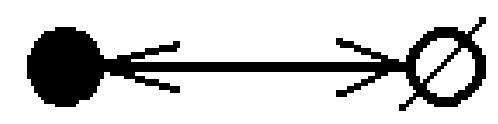

(a)

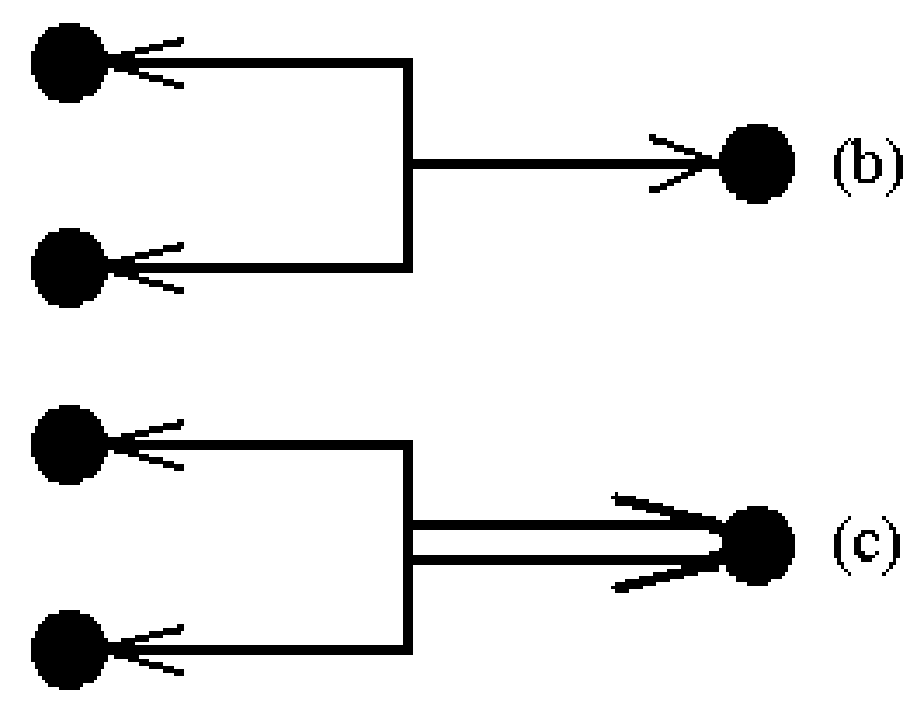

Figure 5: The cycles "putting-out" bifurcation types: a) spontaneous "putting-out"; b) "puttings-out" without any changes of period; c) "putting-out" with the period doubling. 